\title{
Broncoscopia en trasplante pulmonar
}

\author{
Bronchoscopy in pulmonary transplantation
}

Nelson PAEZ EsPinel, MD.

\section{Resumen}

El trasplante pulmonar como opción a varias patologías terminales ha permitido un aumento en la supervivencia de los pacientes que las padecen. La broncoscopia es un procedimiento de rutina realizado antes y después del trasplante que permite la detección temprana de procesos infecciosos, rechazo, bronquiolitis obliterante y alteraciones en la vía aérea como estenosis, dehiscencia de la anastomosis y granulomas. Además de ser un método diagnóstico en algunos casos permite el tratamiento de dichas complicaciones durante el procedimiento con el empleo de endoprótesis, dilatación con balón y suministro de antibióticos.

Palabras clave: trasplante pulmonar, broncoscopia, infección pulmonar, rechazo, complicaciones de la vía aérea.

\begin{abstract}
Lung transplantation as an option in various terminal conditions has increased patient survival. Bronchoscopy is a routine procedure carried out before and after transplantation. It allows for early detection of infectious processes, rejection, bronchiolitis obliterans, and alterations of the airway such as stenosis, anastomotic dehiscence, and granulomas. Besides being a diagnostic method, it sometimes allows for treating such complications during the procedure by way of endoprosthesis, balloon dilatation, and administration of antibiotics.
\end{abstract}

Keywords: lung transplantation, bronchoscopy, lung infection, rejection, airway complications.

El trasplante pulmonar es la alternativa actual para el tratamiento de algunas enfermedades pulmonares irreversibles (1). En los últimos diez años hay un aumento de la supervivencia global, tanto a corto como a largo plazo, si bien los pacientes deben someterse a los riesgos derivados del procedimiento quirúrgico, a las consecuencias de la manipulación de la vía aérea, a los cambios del pulmón trasplantado, así como a aquellos derivados del tratamiento inmunosupresor (2).

La broncoscopia es un procedimiento de rutina tanto en el donante como en el receptor. En el primero se usa para excluir infección y en el segundo para detectar alteraciones de la anastomosis bronquial, para seguimiento del estado del injerto, así como para el diagnóstico de rechazo e infecciones. Es bien tolerada y su tasa de complicaciones no es mayor a la población general (3). Es, además, útil para detectar rechazo agudo, al igual que bronquiolitis linfocítica y obliterante como marcador de rechazo crónico (4).
Especialista en medicina interna y neumología. JEFE DEL ÁREA DE HOSPITALIZACIÓN. Fundación Neumológica Colombiana.

Correspondencia: Nelson Paez Espinel, correo electrónico: npaez@neumologica.org Recibido: 28/10/14, Aceptado: 10/12/14. 
Puede indicarse en forma periódica (broncoscopia de vigilancia) o ante cambios clínicos, para toma de biopsias transbronquiales y lavado broncoalveolar. La periodicidad de realización es variable y depende de cada centro. Mediante comunicaciones personales con médicos del programa de trasplante de Medellín, Canadá, Chile y nuestro centro, se determinó que luego de trasplante los pacientes son programados para broncoscopia en promedio tres a ocho veces en los primeros doce meses, y luego cada año o en caso de cambios clínicos. La variabilidad en el número de procedimientos en cada grupo corresponde al acuerdo en que las broncoscopias de vigilancia aportan información valiosa para el ajuste de la inmunosupresión y la detección de infecciones y complicaciones, en contraposición con los riesgos inherentes a la realización del procedimiento, o a no encontrar diferencia en la sobrevida de pacientes trasplantados si son sometidos a broncoscopia por un protocolo de vigilancia o por indicación clínica y a la experiencia acumulada que ha modificado el número total de broncoscopias a través del tiempo $(5,6)$.

\section{Lavado broncoalveolar}

Se realiza con técnica convencional remitiendo muestras para estudio citológico y de microbiología para coloraciones y cultivos.

La presencia de neutrofilia por encima del 20\% puede indicar rechazo crónico y se relaciona con desarrollo de bronquiolitis obliterante en algunos pacientes (7).

Muchos estudios han medido en lavado broncoalveolar, como marcadores de rechazo y de bronquiolitis obliterante, la elevación de los niveles de interleucina 8,15 y 17, interferón gamma, linfocitos TCD8 y algunas metaloproteinasas. La activación de linfocitos TCD4 y células asesinas naturales, puede deberse a rechazo agudo y crónico. Sin embargo, estos resultados son preliminares, no están validados y aun no pueden utilizarse en la práctica diaria ni reemplazan la biopsia transbronquial (8).

\section{Biopsia transbronquial}

Sigue siendo el estándar de oro en el diagnóstico de rechazo en trasplante pulmonar. No tiene ninguna modificación en la técnica de aplicación ni en el procesamiento. La evaluación sistemática del espécimen a cargo de patólogos con experiencia en este tipo de análisis, es primordial para lograr resultados concluyentes y evitar grandes variabilidades en la interpretación de las mismas $(9,10)$.

\section{Cepillado bronquial}

No está indicado en la actualidad como procedimiento rutinario en pacientes con trasplante pulmonar, excepto en alteraciones de la mucosa sugestivas de neoplasia o infección.

Algunos estudios han explorado la utilidad de estudio con chip de ADN de células bronquiales obtenidas por cepillado bronquial en búsqueda de diferencia en los perfiles genéticos de pacientes con y $\sin$ bronquiolitis obliterante (11).

Así mismo, se ha propuesto que existan perfiles de citoquinas inflamatorias de las células $\mathrm{T}$ intraepiteliales bronquiales, que pueden ser más relevantes que la sangre periférica o las células del lavado broncoalveolar para evaluar el estado inmunológico del injerto (12).

\section{Complicaciones de la vía aérea}

Ocurre hasta en 20 pacientes de cada cien trasplantes. Se estima una mortalidad que no supera el $5 \%$, sin un impacto mayor en la supervivencia global comparable a la de los trasplantados sin estas complicaciones (13). Se dividen según el tiempo de aparición en agudas o crónicas, según se presenten antes o después de tres meses (tabla 1).

Las anastomosis del bronquio es especialmente susceptible a la isquemia ya que solo recibe irrigación retrógrada de la arteria pulmonar las primeras ocho semanas, tiempo necesario para generar circulación colateral de la irrigación bronquial (14). Además, por estar comunicada con el exterior, tiene riesgo de colonización e infección. 
Tabla 1. Complicaciones de la vía aérea después de trasplante pulmonar.

\begin{tabular}{|c|c|}
\hline \multirow[t]{3}{*}{ Estenosis } & - Estenosis de la anastomosis bronquial \\
\hline & - Estenosis segmentaria no anastomótica \\
\hline & - Síndrome del bronquio intermediario evanescente \\
\hline \multirow[t]{4}{*}{ Necrosis / dehiscencia } & - Grado I: sin necrosis \\
\hline & - Grado II: necrosis de mucosa no de pared \\
\hline & - Necrosis de pared menor a $2 \mathrm{~cm}$ de anastomosis \\
\hline & - Necrosis de pared mayor a $2 \mathrm{~cm}$ de anastomosis \\
\hline \multirow[t]{2}{*}{ Granulomas } & - Menor que $50 \%$ de la luz \\
\hline & - Mayor que $50 \%$ de la luz \\
\hline \multirow[t]{2}{*}{ Malacia } & - Difusa \\
\hline & - De la anastomosis \\
\hline \multirow[t]{3}{*}{ Fístula } & - Bronco pleural \\
\hline & - Bronco mediastinal \\
\hline & - Broco vascular \\
\hline \multirow[t]{2}{*}{ Infección } & - Anastomótica \\
\hline & - No anastomótica \\
\hline
\end{tabular}

Se han identificado varios factores de riesgo para las complicaciones de la vía aérea: tiempo de isquemia de órgano, técnica y anastomosis quirúrgica, infecciones postquirúrgicas tempranas, tiempo de ventilación mecánica del donante, disfunción primaria grave del injerto y desproporción de la altura donante-receptor (15). El uso de sirolimus se ha asociado con incidencia alta de complicaciones de la vía aérea y por tanto, no debe usarse antes de la cicatrización completa de la anastomosis.

\section{Estenosis de la vía aérea}

Es la complicación más común luego de trasplante pulmonar y ocurre habitualmente en el primer semestre consecutivo a éste (16).

Habitualmente se detecta durante las broncoscopias de vigilancia o por síntomas (disnea, neumonía o falla respiratoria). En más del $95 \%$ de los casos ocurre en la anastomosis aunque se ha descrito distal a la misma (17); el sitio más frecuente es el bronquio intermediario, y puede desaparecer por completo (síndrome del bronquio intermediario evanescente) (18).

Auque la radiografía del tórax y la tomografía axial computarizada pueden mostrar hallazgos compatibles, la evaluación broncoscópica es el estándar de referencia y deberá practicarse siempre previo a decidir el tratamiento (19), que como en otras lesiones de la vía aérea debe ser multimodal e incluir dilatación con balón, resección con láser o argón plasma e implante de endoprótesis para las cuales se tendrán las mismas consideraciones que en estenosis de la vía aérea de pacientes no trasplantados. Para tal fin se prefieren endoprótesis de silicona o metálicas recubiertas (20).

\section{Dehiscencia de anastomosis}

Es una complicación rara (1\%) cuya mortalidad es alta (21). La detección precoz se hace mediante broncoscopia, ya que los hallazgos por imagen, relacionados con la fuga de aire, casi siempre son tardíos. Debe sospecharse en pacientes con 
neumotórax persistente, neumomediastino o enfisema subcutáneo entre otros. Con menor frecuencia en la tomografía pueden verse colecciones líquidas o hidroaéreas en la pared del bronquio.

Los defectos pequeños (menores de $4 \mathrm{~mm}$ ) tienen mejor pronóstico de recuperación.

Las intervenciones utilizadas incluyen endoprótesis recubiertas, drenajes torácicos para mantener la expansión pulmonar y antimicrobianos profilácticos.

Las complicaciones incluyen infecciones severas, necesidad de reintervención y neumonectomía.

\section{Granulomas}

Se espera que aparezca algo de tejido de granulación en la anastomosis pero en escasa cantidad. La proliferación de tejido con obstrucciones de más del 50\%, se presentará como dificultad respiratoria, neumonía y atelectasia. Hay condiciones que pueden aumentar la granulación como la infección por Aspergillus spp.

El tratamiento incluye desbridamiento cauterización, mitomicina local y braquiterapia, en algunos casos. Es factible la recurrencia (22).

\section{Infección}

Es una complicación y un factor de riesgo para estenosis o granulomas. Las infecciones respiratorias bacterianas de la población general, pueden afectar a estos pacientes con severidad variable y compromiso de todo el árbol bronquial o del área

Tabla 2. Indicaciones de broncoscopia en trasplante pulmonar

\begin{tabular}{|ll|}
\hline 1. & Evaluación del pulmón del donante \\
2. & Broncoscopia de vigilancia periódica \\
3. & Broncoscopia por indicación clínica \\
& a. Rechazo \\
& b. Infección \\
4. Complicaciones de la vía aérea
\end{tabular}

de la anastomosis. La infección más frecuente relacionada con el estado de inmunosupresión de estos pacientes, es producida por Aspergillus spp y casi nunca se torna invasiva. Puede controlarse con antifúngicos y en ocasiones requiere desbridamiento del tejido necrótico bronquial (23).

En conclusión, los pacientes con trasplante pulmonar requieren estudios broncoscópicos para verificar su evolución, ante cambios clínicos y para tratamiento de las diferentes complicaciones. Con base en la publicación de Fernández Bussy y colaboradores en el 2011 (24), puede resumirse así el tratamiento de las complicaciones:

- Las estenosis bronquiales se tratan inicialmente con dilataciones con balón. Generalmente, son varias sesiones y posteriormente se implanta endoprótesis de silicona o híbridas idealmente luego de la octava semana.

- Los granulomas y el tejido de granulación se trata con terapia térmica con electrocauterio, argón plasma o láser.

- Las dehiscencias menores de $4 \mathrm{~mm}$ pueden tratarse con endoprótesis con control cercano y de común acuerdo con cirugía de tórax.

- La broncomalacia severa muy sintomática, debe tratarse con ventilación mecánica no invasiva con presión positiva y, en casos selectos, con endoprótesis.

\section{Bibliografía}

1. Yusen RD, Edwards LB, Kucheryavaya AY, Benden C, Dipchand AI, Dobbels F. International Society for Heart and Lung Transplantation. The registry of the International Society for Heart and Lung Transplantation: thirty-first adult lung and heart-lung transplant report-2014. J Heart Lung Transplant. 2014;33(10):1009-24.

2. Laporta Hernández R, Lázaro Carrasco MT, Varela de Ugarte A, Ussetti Gil P. Long-term follow-up of the lung transplant patient. Arch Bronconeumol. 2014;50(2):67-72.

3. Trulock EP, Ettinger NA, Brunt EM, Pasque MK, Kaiser LR, Cooper JD. The role of transbronchial lung biopsy in the treatment of lung transplant recipients. An analysis of 200 consecutive procedures. Chest. 1992;102:1049-54. 
4. Sandrini A, Glanville AR. The controversial role of surveillance bronchoscopy after lung transplantation. Curr Opin Organ Transplant. 2009;14(5):494-8.

5. Valentine VG, Gupta MR, Weill D, Lombard GA, LaPlace SG, Seoane L. Single-institution study evaluating the utility of surveillance bronchoscopy after lung transplantation. J Heart Lung Transplant. 2009;28(1):14-20.

6. Glanville AR. The role of surveillance bronchoscopy post-lung transplantation. Semin Respir Crit Care Med. 2013;34(3):414-20.

7. Neurohr C, Huppmann P, Samweber B, Leuschner S, Zimmermann G, Leuchte H. Munich Lung Transplant Group. Prognostic value of bronchoalveolar lavage neutrophilia in stable lung transplant recipients. J Heart Lung Transplant. 2009;28(5):468-74.

8. Snell GI, Boehler A, Glanville AR, McNeil K, Scott JP, Studer SM. Eleven years on: a clinical update of key areas of the 1996 lung allograft rejection working formulation. J Heart Lung Transplant. 2007;26(5):423-30.

9. Arcasoy SM, Berry G, Marboe CC, Tazelaar HD, Zamora MR, Wolters HJ, et al. Pathologic interpretation of transbronchial biopsy for acute rejection of lung allograft is highly variable. Am J Transplant. 2011;11:320-8.

10. Bhorade SM, Husain AN, Liao C, Li LC, Ahya VN, Baz MA, et al. Interobservador variability in grading transbronquial lung biopsy specimens after lung transplantation. Chest. 2013;143:1717-24.

11. Skawran B, Dierich M, Steinemann D, Hohlfeld J, Haverich A, Schlegelberger B. Bronchial epithelial cells as a new source for differential transcriptome analysis after lung transplantation. Eur J Cardiothorac Surg. 2009;36(4):715-21.

12. Hodge G, Hodge S, Reynolds PN, Holmes M. Compartmentalization of intracelular proinflammatory cytokines in bronchial intraepithelial $\mathrm{T}$ cells of stable lung transplant patients. Clin Exp Immunol. 2006;145(3):413-9.

13. Meyers BF, de la Morena M, Sweet SC, Trulock EP, Guthrie TJ, Mendeloff EN. Primary graft dysfunction and other selected complications of lung transplantation: a single-center experience of 983 patients. J Thorac Cardiovasc Surg. 2005;129:1421-9.
14. Murthy SC, Blackstone EH, Gildea TR, Gonzalez-Stawinski GV, Feng J, Budev M. Impact of anastomotic airway complications after lung transplantation. Ann Thorac Surg. 2007;84:401-9.

15. Van De Wauwer C, Van Raemdonck D, Verleden GM, Dupont L, De Leyn P, Coosemans W. Risk factors for airway complications within the first year after lung transplantation. Eur J Cardiothorac Surg. 2007;31:703-10.

16. Krishnam MS, Suh RD, Tomasian A, Goldin JG, Lai C, Brown K. Postoperative complications of lung transplantation: radiologic findings along a time continuum. Radiographics. 2007;27:957-74.

17. Hasegawa T, Iacono AT, Orons PD, Yousem SS. Segmental nonanastomotic bronchial stenosis after lung transplantation. Ann Thorac Surg. 2000;69:1020-4.

18. Lari SM, Gonin F, Colchen A. The management of bronchus intermedius complications after lung transplantation: a retrospective study. J Cardiothorac Surg. 2012;7:8-138.

19. Allah MF, Hussein SR, El-Asmar AB, Zoair HM, Mohamed GA, Metwaly AM. Role of virtual bronchoscopy in the evaluation of bronchial lesions. J Comput Assist Tomogr. 2012;36(1):94-9.

20. Folch E, Mehta AC. Airway interventions in the tracheobronchial tree. Semin Respir Crit Care Med. 2008;29:441-52.

21. Usuda K, Gildea T, Pandya C, Mehta AC. Bronchial dehiscence. J Bronchol. 2005;12:164-5.

22. Herrera JM, McNeil KD, Higgins RS, Coulden RA, Flower $\mathrm{CD}$, Nashef SA. Airway complications after lung transplantation: treatment and long-term outcome. Ann Thorac Surg. 2001;71:989-3

23. Bhaskaran A, Hosseini-Moghaddam SM, Rotstein C, Husain S. Mold infections in lung transplant recipients. Semin Respir Crit Care Med. 2013;34(3):371-9.

24. Fernández-Bussy S, Majid A, Caviedes I, Akindipe O, Baz M, Jantz M. Treatment of airway complications following lung transplantation. Arch Bronconeumol. 2011;47(3):128-33. 